\title{
NOUVELle
}

\section{Les sentiments naissent sous des bonnes étoiles, ou comment les astrocytes contrôlent nos émotions}

Angel Baudon, Alexandre Charlet
CNRS et Université de Strasbourg, Institut des neurosciences cellulaires et intégratives (INCI, UPR3212), 8 allée du Général Rouvillois, 67000 Strasbourg, France. acharlet@unistra.fr
Le cerveau : des neurones...

Le cerveau est le siège de la pensée, des émotions, et de la gestion de nos réactions, normales ou pathologiques, en réponse à notre environnement. Cet organe est composé de plusieurs centaines de milliards de cellules. Parmi elles, les neurones ont particulièrement attiré le regard de la recherche et de la médecine en raison de leur activité électrique. Cette activité est à l'origine d'un codage binaire, en apparence assez simple, qui a donné beaucoup d'espoirs aux neuroscientifiques cherchant à décrypter le code neuronal dans le but de comprendre nos comportements les plus complexes, une approche qui a d'ailleurs connu quelques succès spectaculaires au cours des dernières décennies.
... et des cellules gliales, parmi lesquelles les astrocytes

Cependant, le cerveau possède presqu'autant de cellules gliales que de neurones [1], ainsi nommées car elles ont d'abord été considérées comme de la glu sans autre rôle que celui de soutien pour les neurones. Mais les vingt dernières années ont vu s'accumuler de nombreuses preuves que les cellules gliales sont aussi des acteurs du traitement de l'information dans le cerveau. Parmi ces cellules, les astrocytes, en particulier, collaborent étroitement avec les neurones, dont ils modifient directement l'activité [2]. Des substances neuroactives détectées par les astrocytes recrutent ainsi des voies de signa- lisation qui entraînent des variations de la concentration intracellulaire des ions calcium, permettant à ces cellules de moduler l'activité du réseau neuronal environnant. S'il est désormais établi que les neurotransmetteurs classiques, tels que le glutamate ou l'adrénaline, peuvent déclencher une signalisation calcique dans les astrocytes [2], les effets potentiels des neurohormones sur ces cellules restent, quant à eux, mal connus.

Nous avons étudié les effets de l'ocytocine sur les astrocytes chez deux rongeurs [3] : la souris (Mus musculus) et le rat (Rattus norvegicus), génétiquement plus proche de nous que ne l'est la souris [4]. 

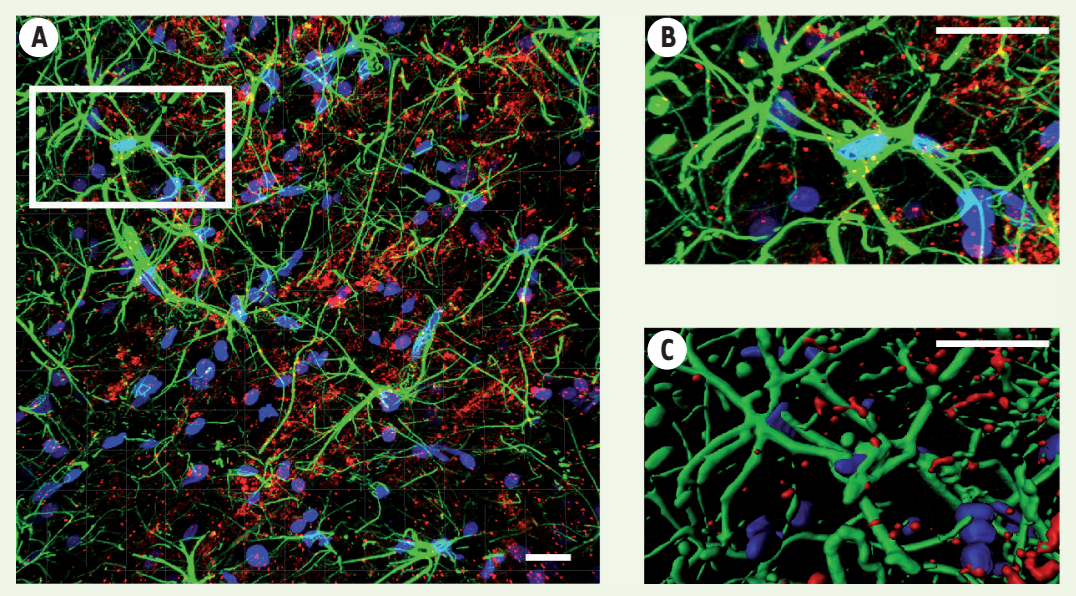

Figure 1. Les astrocytes de l'amygdale expriment le récepteur de l'ocytocine. (A, B) Images de microscopie confocale en plan large $(\boldsymbol{A})$ et détail d'un astrocyte $(B)$. Les astrocytes sont révélés par marquage de la glutamine synthase (en vert), et l'ARN messager codant le récepteur de l'ocytocine par une fluorescence rouge. Les noyaux cellulaires sont révélés par une fluorescence bleue (DAPI). (C) Reconstruction tridimensionnelle permettant une quantification spatiale de la structure cellulaire et des marqueurs utilisés. Barre d'échelle : $10 \mu \mathrm{m}$.

\section{L'ocytocine module l'activité des astrocytes}

L'ocytocine, un neuropeptide de 9 acides aminés, est connu pour son rôle dans le contrôle des émotions (douleur, anxiété) et dans les interactions sociales, et plus généralement comme promoteur du bien-être [5-7]. On pensait jusqu'alors que les effets de l'ocytocine impliquaient uniquement son action directe sur les neurones. Or, nous avons montré que certains astrocytes de l'amygdale, une structure cérébrale impliquée dans le contrôle des émotions, expriment le récepteur de l'ocytocine (Figure 1). Ces astrocytes présentent des caractéristiques distinctes: ils sont plus grands que leurs voisins, possèdent plus de ramifications, et sont en contact avec de nombreux astrocytes dépourvus de ce récepteur. L'imagerie dynamique du calcium intracellulaire a confirmé que l'ocytocine active directement de très nombreux astrocytes de l'amygdale, et que ces derniers peuvent transmettre l'information aux astrocytes voisins grâce à leurs jonctions communicantes (gap junctions) (Figure 2).
Du circuit neuronal au comportement : les astrocytes relayent le message

\section{ocytocinergique}

L'analyse électrophysiologique du réseau neuronal de l'amygdale a indiqué que les astrocytes de l'amygdale activés par l'ocytocine pourraient sécréter un neuromodulateur, la D-sérine, qui favoriserait la neurotransmission glutamatergique via les récepteurs NMDA ( $\mathrm{N}$-méthyl-D-aspartate) du glutamate, et ainsi augmenter l'activité des neurones environnants (Figure 2). Les astrocytes joueraient donc un rôle actif dans la modulation de l'activité du réseau neuronal de l'amygdale [2]. Cette modulation a été observée dans l'amygdale centrale, où l'effet anxiolytique de l'ocytocine avait précédemment été en partie élucidé [6] $(\rightarrow)$.

$(\rightarrow)$ Voir la Nouvelle de A. Baudon et Nous avons étudié A. Charlet, $m / s n^{\circ} 1$, janvier 2020, page 9 l'implication comportementale de l'activation du réseau astro-neuronal de l'amygdale en modifiant son activité par différentes techniques. Chez certains animaux, les astrocytes de l'amygdale ont été activés par une technique optogénétique, tandis que chez d'autres, le récepteur de l'ocy- tocine a été supprimé dans les astrocytes, empêchant ainsi leur activation directe par l'ocytocine. Les animaux ont ensuite été soumis à des tests comportementaux de nociception (perception sensorielle de la douleur), d'évaluation de l'anxiété, ou de valence émotionnelle, souvent associée à l'aspect émotionnel de la douleur [8, 9]. II apparaît que si la nociception semble peu impactée par l'activation du réseau astroneuronal de l'amygdale par l'ocytocine, cette dernière induit en revanche une forte diminution de l'anxiété et une nette préférence de place ${ }^{l}$, témoin d'une modification de la valeur émotionnelle accordée à l'environnement (Figure 2). Ainsi, une situation stressante semblera moins anxiogène, une douleur paraîtra moins importante, et la sensation de bien-être sera renforcée.

\section{Perspectives}

Nous avons donc montré, dans deux espèces de rongeurs, que la libération d'ocytocine au sein de l'amygdale agit directement sur les astrocytes, dont l'activation provoque à son tour une augmentation de l'activité neuronale se traduisant par une diminution des comportements anxieux. Cette découverte étaye la théorie selon laquelle les neurones et les astrocytes constitueraient des canaux de communication ayant des cinétiques différentes: les influx électriques portés par les neurones sont brefs et rapides (de la milliseconde à la seconde), tandis que les signaux calciques produits par les astrocytes sont longs et lents (de la seconde à plusieurs dizaines de minutes). Ainsi, le sentiment diffus et prolongé de bien-être suivant la libération d'ocytocine peut être expliqué par le recrutement d'un réseau astrocytaire au sein de l'amygdale.

\footnotetext{
Le test de «préférence de place» (conditioned place preference, (PP) permet de tester la valence émotionnelle d'un stimulus. Le dispositif expérimental comporte une arène contenant deux chambres. L'animal reçoit une stimulation (injection d'un agent pharmacologique, stimulation optique, etc.) dans un des deux compartiments puis, lors de la phase de test, on observe si l'animal préfère passer du temps dans le compartiment où il a reçu le stimulus, ou dans l'autre (où il a par exemple reçu un placebo dans le cas de l'injection d'une solution).
} 


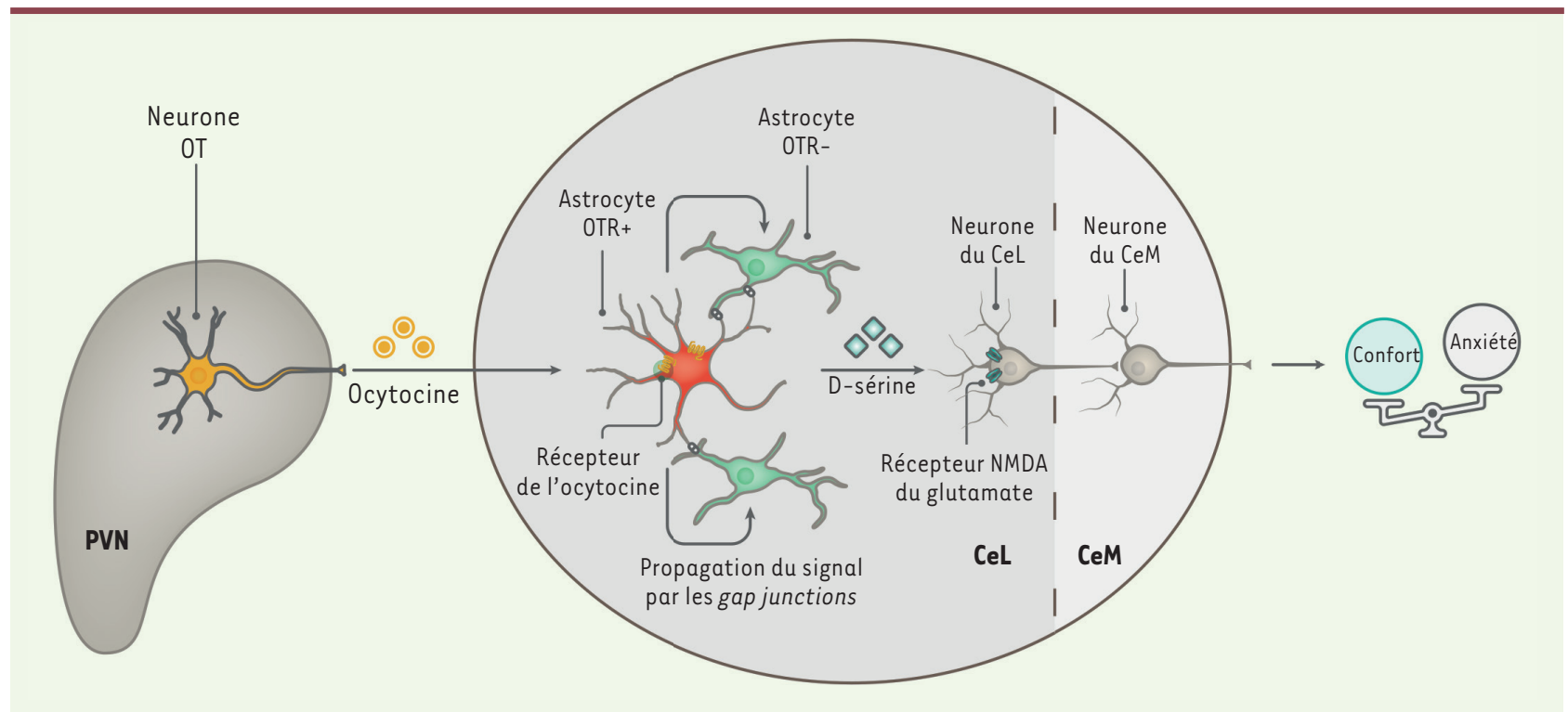

Figure 2. Schéma récapitulatif des effets de l'ocytocine sur le réseau astro-neuronal de l'amygdale centrale. L'ocytocine (OT) synthétisée par certains neurones du noyau paraventriculaire de l'hypothalamus (PVN) est sécrétée au sein de l'amygdale centrale latérale (CeL). Là, elle se lie à son récepteur (OTR) présent notamment sur les astrocytes (en rouge), ce qui provoque une augmentation de la concentration intracellulaire des ions calcium. Ce signal est transmis aux astrocytes voisins (en vert) à travers les jonctions communicantes (gap junctions) entre ces cellules. Cette activation du réseau astrocytaire libère la D-sérine, qui agit sur les récepteurs NMDA du glutamate neuronaux, facilitant l'activation des neurones du CeL, et aboutissant à l'inhibition des neurones de l'amygdale centrale médiane (CeM). La modulation de ce réseau astro-neuronal par I'OT diminue l'anxiété et induit une sensation de confort.

On sait que l'anxiété généralisée et la dépression accompagnent fréquemment les douleurs chroniques, qui touchent aujourd'hui près de $20 \%$ de la population [10]. Or, nos résultats montrent que l'activation d'une population astrocytaire spécifique par l'ocytocine affecte l'un des aspects les plus délétères de la douleur chronique: son impact sur la sphère émotionnelle. En ce sens, ces résultats sont prometteurs, car ils permettent d'imaginer de nouveaux traitements de la douleur chronique, ciblant les cellules gliales et, potentiellement, sans effet indésirable. $\diamond$

Reaching for the stars: How astrocytes regulate positive emotions via oxytocin

\section{LIENS D'INTÉR̂̂T}

Les auteurs déclarent n'avoir aucun lien d'intérêt concernant les données publiées dans cet article.

\section{RéFÉRENCES}

1. Herculano-Houzel S. The glia/neuron ratio: how it varies uniformly across brain structures and species and what that means for brain physiology and evolution. Glia $2014 ; 62$ : 1377-91.

2. Khakh BS, Sofroniew MV. Diversity of astrocyte functions and phenotypes in neural circuits. Nat Neurosci 2015 ; 18 : 942-52

3. Wahis J, Baudon A, Althammer F, et al. Astrocytes mediate the effect of oxytocin in the central amygdala on neuronal activity and affective states in rodents. Nat Neurosci 2021 ; 24 : 529-41.

4. Rat Genome Sequencing Project Consortium. Genome sequence of the Brown Norway rat yields insights into mammalian evolution. Nature 2004 ; 428 : 493-521.

5. Eliava M, Melchior M, Knobloch-Bollmann HS, et al. A new population of parvocellular oxytocin neurons controlling magnocellular neuron activity and inflammatory pain processing. Neuron $2016 ; 89$ : 1291-304.

6. Baudon A, Charlet A. Un engramme ocytocinergique pour apprendre et contrôler sa peur. Med Sci (Paris) $2020 ; 36: 9-11$

7. Tang Y, Benusiglio D, Lefevre A, et al. Social touch promotes interfemale communication via activation of parvocellular oxytocin neurons. Nat Neurosci 2020 ; $23: 1125-37$.

8. Veinante P, Yalcin I, Barrot M. The amygdala between sensation and affect: a role in pain. J Mol Psychiatry $2013 ; 1: 1-9$

9. King T, Vera-Portocarrero L, Gutierrez T, et al. Unmasking the tonic-aversive state in neuropathic pain. Nat Neurosci 2009 ; 12 : 1364-6.

10. Woo AK. Depression and anxiety in pain. Reviews in Pain $2010 ; 4: 8-12$

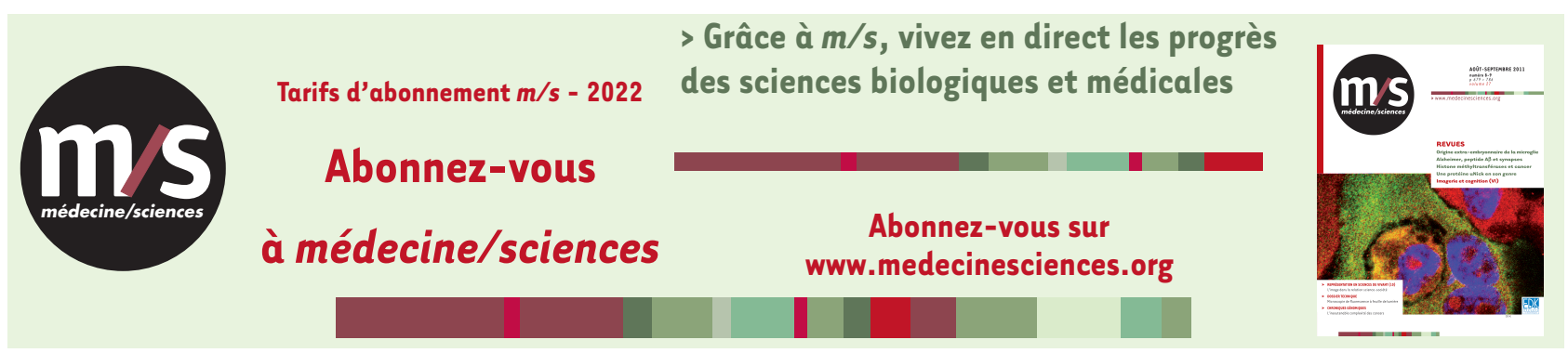

